\title{
Moralstrukturen professionellen Handelns
}

\author{
Heinz Messmer
}

Zusammenfassung: Der nachfolgende Beitrag befasst sich mit Erscheinungsformen von Moral in der Beziehung zwischen Professionellen und AdressatInnen der Sozialen Arbeit. Ausgangspunkt der Analysen ist eine Sicht auf Moral, die ihren Gegenstand als Kommunikation von (Miss-)Achtung betrachtet. Am Fall der Konversationsanalyse von Hilfeplangesprächen wird gezeigt, dass Moral in den Interaktionen zwischen Professionellen und KlientInnen allgegenwärtig ist und die Integration der Beteiligten in das Hilfeplansetting unterstützt. Probleme im Umgang mit Moral entstehen dagegen regelmäßig im Kontext der Fallkonstitution, insbesondere bei der Erörterung eines die Hilfe auslösenden Defizits. Hier sieht sich das professionelle Handeln mit strukturell widersprüchlichen Handlungsanforderungen konfrontiert, die auf das Spannungsfeld institutioneller und interaktiver Funktionsbestimmungen in der Sozialen Arbeit verweisen.

Schlüsselwörter: Moral · Professionelles Handeln · Kommunikation und Interaktion in der Sozialen Arbeit · Hilfeplanung · Konversationsanalyse

\section{Moral structures of professional action}

\begin{abstract}
The following article deals with manifestations of morality in the relationship between professionals and users of social work. The starting point of this investigation is a view of morality that sees morality as the communication of (mis-)respect. Using conversation analyses of the care planning conferences it will be shown that morality is ubiquitous in the interactions between professionals and clients, and supports the integration of those involved in the care planning setting. Problems regularly arise in dealing with the constitution of the case, especially in relation of the help-causing deficit. Here the professional acting is confronted with structurally conflicting demands, pointing to the mismatch of institutional and interactive functions of social work.
\end{abstract}

Keywords: Ethics - Professional behavior - Communication and interaction in social work Care planning $\cdot$ Conversation analysis

(c) VS Verlag für Sozialwissenschaften 2012

Prof. Dr. H. Messmer (ه)

Institut Kinder- und Jugendhilfe, Fachhochschule Nordwestschweiz,

Hochschule für Soziale Arbeit, Thiersteinerallee 57, 4053 Basel, Schweiz

E-Mail: heinz.messmer@fhnw.ch 


\section{Soziale Arbeit als moralische Disziplin}

Theorie und Praxis der Sozialen Arbeit sind in hohem Maße moralisch fundiert (vgl. Fuchs 2004; Thiersch 2011). Als eine im Grundsatz ,helfende Disziplin“ werden ihr vorwiegend positiv konnotierte Funktionen und Aufgaben zugeschrieben, die zur Verbesserung der Lebenssituation ihrer Klientel beitragen sollen. Auch wenn ihre identitätsprägenden Semantiken (Hilfe, Fürsorge, Wohlfahrt, Daseinsnachsorge, soziale personenbezogene Dienstleistungen, u. ä.m.) mit der Zeit variieren und kontinuierlich den jeweiligen wohlfahrtsstaatlichen Entwicklungsdynamiken angepasst werden, ist ihre symbolische Aussagekraft dennoch nahezu unverändert geblieben. Nach wie vor erscheint „Hilfe“ gegenüber „Nicht-Hilfe“ als ein normativ vorzugswürdiger Wert, was sich allein schon daran zeigt, dass Erstere sich im Unterschied zur Letzteren nicht eigens zu rechtfertigen braucht.

Selbst wenn die beruflichen Motive des Helfens in der Gründerzeit von Erziehungswissenschaft und Sozialpädagogik (,selbstlose Hilfe“ (Flittner), (christlich motivierte) Nächstenliebe und Barmherzigkeit, ,erzieherische Hingabe an den einzelnen Menschen“ (Nohl), „Trieb zur Hingabe an den anderen“ (Spranger), vgl. Gängler 2011) aus heutiger Sicht pathetisch und anachronistisch erscheinen, sind die ethischen Anforderungen an das professionelle Handeln auch heute noch gültig. So lassen sich aus dem Grundrecht eines jungen Menschen ,auf die Förderung seiner Entwicklung und auf Erziehung zu einer eigenverantwortlichen und gemeinschaftsfähigen Persönlichkeit“ (§1 Abs. 1 SGB VIII, Wiesner 2000) weitreichende Rückschlüsse auf das professionelle Handeln derjenigen ziehen, die für die Leistungserbringung zuständig sind - etwa im Hinblick auf den Abbau von Benachteiligungen oder den Schutz von Kindern und Jugendlichen vor Gefahren für ihr Wohlergehen. Darüber hinaus werden mit fortschreitender Professionalisierung vermehrt Verhaltensmaximen für das fachliche Handeln formuliert: „Die Praxis der Sozialen Arbeit ist ethisch begründet, wenn das Handeln aufgrund ihrer moralischen Kriterien sowie ihrer professionellen Grundsätze reflektiert wird“, formuliert beispielsweise der „Berufskodex Soziale Arbeit Schweiz“ (vgl. AvenirSocial 2010). Darunter fällt u.a. „die Grundhaltung der streitbaren Toleranz, die Verpflichtung, die Würde des Menschen $\mathrm{zu}$ achten und $\mathrm{zu}$ verteidigen und dabei auch für die soziale Gerechtigkeit einzutreten“ (vgl. Heiner 2007, S. 171). Schließlich fällt auch der starke Zuwachs an Reflexionsangeboten auf, der sich speziell über die moralische Fundierung der Sozialen Arbeit begründet (vgl. Gruber 2005; Kuhrau-Neumärker 2005; Dungs et al. 2006; Eisenmann 2006; Friesinger 2007; Lob-Hüdepohl 2007; Martin 2007).

Ebenso wie in den klassischen Professionen des Rechts, der Medizin, Religion, etc. haben es die Fachkräfte der Sozialen Arbeit mit Personen zu tun, die in zentralen Bereichen ihres Lebens Probleme erfahren, für die es oft keine alltäglichen Lösungen gibt (vgl. Hughes 1965; Stichweh 2004). Für die Betroffenen entsteht daraus eine spezifische Form der Abhängigkeit: Sie benötigen Wissen, Kompetenzen und/oder Ressourcen, über die sie selbst nicht verfügen und dadurch in zentralen Lebensbereichen von den Unterstützungsleistungen anderer abhängig werden. Soll dieses strukturell angelegte Machtungleichgewicht den Betroffenen nicht zum Nachteil gereichen, bedarf es Regeln einer fachlich zulässigen Einflussnahme und Instrumenten handlungsbezogener Selbstreflexion, die vor missbräuchlichen Formen der Machtausübung schützen. Entsprechend unterscheidet sich 
die Ethik der Sozialen Arbeit nicht grundsätzlich von der einer anderen Profession: Sofern professionelles Handeln durch hohe Ermessensspielräume gekennzeichnet ist, sind Reflexivität und moralisches Bewusstsein notwendige Dimensionen seiner Begrenzung.

\section{Moral}

Moral ist eine ebenso faszinierende wie schwierige Kategorie. Im Sinne ethischer Verhaltensmaximen besitzt sie nahezu Gesetzeskraft, empirisch zu fassen ist sie gleichwohl nur schwer. Grundlegende Voraussetzungen für die Beurteilung moralischer Qualitäten sind Freiheit und Verantwortlichkeit: Eine Person, die nicht so handelt, wie sie hätte handeln sollen, hätte zumindest so handeln können müssen (vgl. Goffman 1971). Somit stützt sich die Beurteilung moralischer Qualitäten auf ein ideelles Konstrukt. Am greifbarsten wird Moral an den äußersten Polen der Handlungsbewertung (gut/schlecht bzw. gut/böse), wo sich die Qualität sozialer Beziehungen noch am deutlichsten zeigt: Wer beispielsweise dem Freund die Geldbörse stiehlt, handelt nicht nur der „Objektivität des Sollens“ (Jaspers 1973, S. 344) zuwider, sondern geht zudem das Risiko ein, dass seine Identität in den Augen der anderen dauerhaft Schaden nimmt.

Jenseits solcher Extrempositionen zeigt sich Moral dagegen weit weniger klar. Sind weder Regelverstöße noch Verhaltensauffälligkeiten zu erkennen, besteht kaum Anlass für ein moralisches Räsonnement. Explizit moralisch gefärbte Urteile werden mit gutem Grund zudem nur in Ausnahmesituationen kommuniziert, denn sie sind in mehrfacher Weise riskant: Weil Moral auch auf affektive Komponenten des Erlebens reagiert (Empörung, Groll, Scham, etc.), gerät die rationale Auseinandersetzung um moralische Urteile oftmals zu einem schwierigen Unterfangen. Erschwerend tritt hinzu, dass die Kommunikation moralischer Urteile zu Pauschalisierungen neigt und aus einzelnen Handlungen Rückschlüsse auf die Person bzw. ihren Charakter zieht. Luhmann (1978, S.54, ders. 1989) spricht in diesem Zusammenhang von den polemogenen, den Streit auslösenden und eskalierenden Eigenschaften der Moral, sofern sie die Identität des anderen mit einem Stigma bedroht. Paradoxerweise ist die Moralkommunikation gerade auch in moralischer Hinsicht ein fragwürdiges Unterfangen.

Aus soziologischer Sicht ist das Alltagsleben dennoch weit mehr mit moralischen Urteilen verwoben, als ein erster Blick dies vermuten lässt. Bei Simmel (1892/1893) beispielsweise ist von einer unübersehbaren Fülle von Moralprinzipien die Rede, deren Funktion u.a. darin besteht, dass sie den Gegensatz zwischen Egoismus und Altruismus überbrücken. Durkheim (1973, 1976, S. 156 ff.) wiederum sieht im ungezügelten Anwachsen moralischer Imperative die Gefahr, dass die Anforderungen an die soziale Regelbefolgung ab einem bestimmten Grad nicht mehr einlösbar sind. Weber (1934/2010) dagegen behandelt Moral nicht nur als Sachverhalt, in dem sich das gemeinschaftliche Wert- und Sittengesetz manifestiert, sondern der auch gesellschaftsstrukturelle Entwicklungsimpulse begründet. Luhmann (1984, S. 317 ff.) verortet den Sinn der Moral ähnlich wie Simmel in der Vermittlung zwischenmenschlicher und sozialer Interpenetration, die dafür sorgt, dass sich der Einzelne den interaktiven und systemischen Ordnungsvorstellungen unterwirft. Mit Blick auf das Alltagshandeln schließen sich Bergmann und Luckmann (1999) dieser Sichtweise an, obgleich nach ihrem Dafürhalten die ,unsichtbare 
Allgegenwart der Moral“ (Bergmann und Luckmann 1999, S. 13) gegenwärtig in einer entschärften Form und mit zurückgenommenen Geltungsansprüchen agiert.

Auch die Moralphilosophie der Neuzeit sieht den Sinn moralischen Handelns darin begründet, dass es soziale Inklusion unterstützt. Wer sich anderen gegenüber gerecht, hilfsbereit oder altruistisch verhält, stärkt das gemeinschaftliche Zusammenleben in nachhaltiger Weise (vgl. Tarkian 2009; Hörster 2011). „Gut“ ist demnach, ,was die Einheit von individuellem und sozialen Leben befördert" (Beetz 2009, S. 256). Allerdings stellt sich dabei immer auch die Frage nach dem Kontextbezug: So können sich die moralischen Handlungsprinzipien einer Gemeinschaft von Gefängnisinsassen von denen außerhalb der Gefängnismauern stark unterscheiden. Es entsteht das Problem, ob und inwieweit Moral überhaupt moralfrei analysiert werden kann. Vor diesem Hintergrund hilft schließlich der systemtheoretische Moralbegriff weiter. Luhmann (1978) schlägt vor, Moral als ein Achtungserweis zu begreifen, der auf das Gelingen perspektivisch integrierter Kommunikation reagiert. Mit dem Achtungserweis wird honoriert, dass „ein anderer den Erwartungen entspricht, die man für die Fortsetzung sozialer Beziehungen voraussetzen zu müssen meint" (Luhmann 1984, S.318). Diese Vorstellung von Moral bietet den Vorteil, dass sie nicht notwendigerweise auf idealtypische Vorstellungen von „gut“" oder ,böse“" zurückgreifen muss. An die Stelle einer ontologisch vorgängigen Sollensbegründung (Sittengesetz) tritt stattdessen eine stärker prozessorientierte Vorstellung von Moral, die nach den ,faktisch praktizierten Bedingungen wechselseitiger Achtung oder Missachtung" (Luhmann 1978, S. 51) fragt und ihre empirischen Haltepunkte dabei in den faktisch ablaufenden Kommunikationen gelebter Beziehungen findet.

Mit Blick auf die Praxis der Sozialen Arbeit eröffnet die Luhmannsche Vorstellung von Moral eine ebenso anschlussfähige wie empirisch umsetzbare analytische Position: Inhaltlich anschlussfähig ist diese Sichtweise in dem Maße, wie sie den ethischen Handlungsverpflichtungen Sozialer Arbeit (Wertschätzung, Achtsamkeit, Schutz der Würde des Menschen, etc.) nahekommt. Anknüpfungspunkte ihrer empirischen Umsetzbarkeit ergeben sich dagegen durch die These ihrer Einbettung in Kommunikation: Sofern Moral allgegenwärtig ist, müssen sich in den Interaktionen zwischen Professionellen und Klienten ebenfalls Spuren davon zeigen.

\section{Fragestellung und Methode}

Mit den nachfolgenden Analysen ist intendiert, Erscheinungsformen von Moralität in den Interaktionen der Sozialen Arbeit herauszuarbeiten und in ihren Konsequenzen exemplarisch sichtbar zu machen. Die Daten, auf die wir uns dabei beziehen, stammen aus einer konversationsanalytischen Untersuchung zu Aushandlungsprozessen im Hilfeplangespräch (vgl. Messmer und Hitzler 2011). Auf den Untersuchungskontext dieser Studie wird hier nur insoweit eingegangen, als zum Verständnis der herangezogenen Daten unbedingt notwendig ist. Im Einzelnen bezogen sich die Hilfeplangespräche auf Einstieg, Fortschreibung oder Beendigung einer Hilfe im Rahmen der stationären Fremdunterbringung. Merkmale und Besonderheiten der Gesprächsdaten werden an entsprechender Stelle beschrieben. 
Vorausgeschickt seien dagegen einige Anmerkungen zur Methode. Die der Konversationsanalyse zugrundeliegende Haltung geht von der Annahme aus, dass soziale Wirklichkeit als ein Produkt kommunikativer Aktivitäten (Interaktionen) verstanden und in dieser Form auch rekonstruiert werden kann. Was sich demnach als „Wirklichkeit“" sozial bedeutsam erweist, ist auf die Kommunikation wenigstens zweier Individuen angewiesen, die mittels ihrer Äußerungen dem je eigenen Wirklichkeitsverständnis Ausdruck geben und dabei versuchen, ihre Absichten und Motive untereinander zu koordinieren (vgl. Hitzler und Messmer 2011). Aus Sicht der Konversationsanalyse existieren die Sachverhalte sozialer Wirklichkeit daher nicht schon per se, sondern diese besitzen nur insoweit Geltung, wie Individuen sich darauf beziehen und mit anderen darüber kommunizieren.

Entsprechendes gilt für die institutionelle Wirklichkeitsproduktion. Hilfeplanung steht begrifflich für eine Aktivität, mit der sich verschiedene Akteure auf ein gemeinsam hergestelltes Wirklichkeitsverständnis beziehen, um auf dieser Grundlage füreinander nachvollziehbar und einvernehmlich über den weiteren Fortgang der Hilfe zu entscheiden. Das so hergestellte Wirklichkeitsverständnis ist insoweit kontingent, als es auch anders hätte ausfallen können.

Diese Auffassung von Wirklichkeit prägt nicht zuletzt auch die methodische Herangehensweise dieser Methode. Als empirische Datengrundlagen gelten ihr ausschließlich „natürliche“, d.h. von Forschenden unbeeinflussten Kommunikationen. Diese werden mittels Audio-Aufzeichnungen dokumentiert, gemäß konventionellen Transkriptionsregeln (GAT, vgl. Selting et al. 2009) verschriftet und nach datengeleiteten Relevanzkriterien analysiert. Damit wird nicht zuletzt der Flüchtigkeit sozialer Wirklichkeit (vgl. Bergmann 1985) entgegengewirkt, was es gestattet, vor allem die unbemerkten, als selbstverständlich erachteten, unhinterfragten, gleichwohl sinngenerierenden Bausteine sozialer Wirklichkeit einer ausführlichen Analyse zu unterziehen (vgl. Messmer 2012).

\section{Funktion und Formen moralischen Handelns}

Die Untersuchungen in diesem Abschnitt nehmen beispielhaft moralische Phänomene von Hilfeplangesprächen in den Blick. Im Mittelpunkt der nachfolgenden Analysen stehen Fragen zur Allgegenwart der Moral, ebenso wie Fragen zur Vermeidung moralischer Urteile aus Gründen der Moralität sowie die Konfliktanfälligkeit der Moral. Die Analysen stützen sich aus Illustrationsgründen vornehmlich auf Gesprächsdaten aus dem Hilfeplangespräch ,Janine‘. Das hat den Vorteil, dass der Interaktionskontext der einzelnen Analysen überschaubar bleibt und somit die Nachvollziehbarkeit der Dateninterpretation erleichtert. Entsprechend erheben die vorliegenden Analysen zwar keinen Anspruch auf analytische oder systematische Vollständigkeit, verweisen gleichwohl aber auf eine potentielle Verallgemeinerbarkeit der bis dahin gewonnen Einsichten.

\subsection{Moral als Medium der Interaktion}

In diesem Abschnitt wird zunächst die Allgegenwart der Moral als Medium der Interaktion reflektiert. Als Suchkriterium dient uns der Begriff der Achtungskommunikation, die auf das Gelingen perspektivisch integrierter Kommunikation reagiert. Die nachfol- 
gende Analyse nimmt Bezug auf die Eingangssequenz aus dem Hilfeplangespräch (HPG) ,Janine‘. Bei Janine handelt es sich um ein zum Zeitpunkt des Gesprächs 13-jähriges Mädchen, das sich zunehmend der Obhut der Mutter entzieht, wobei die Fachkraft des Jugendamts ein Abgleiten in die Prostituiertenszene befürchtet, nachdem Janine wiederholt mit männlichen Erwachsenen über Nacht weggeblieben ist. Auf Antrag der Mutter wird Janine in der Clearingstelle der Heimeinrichtung XY untergebracht. Das HPG erfolgt etwa 14 Tage danach. Das folgende Exzerpt gibt den Beginn der Aufzeichnung wieder, in dem sich die anwesenden Personen einander vorstellen, bevor der Erziehungsleiter, der das Gespräch moderiert, zum Thema der Sitzung überleitet. ${ }^{1}$

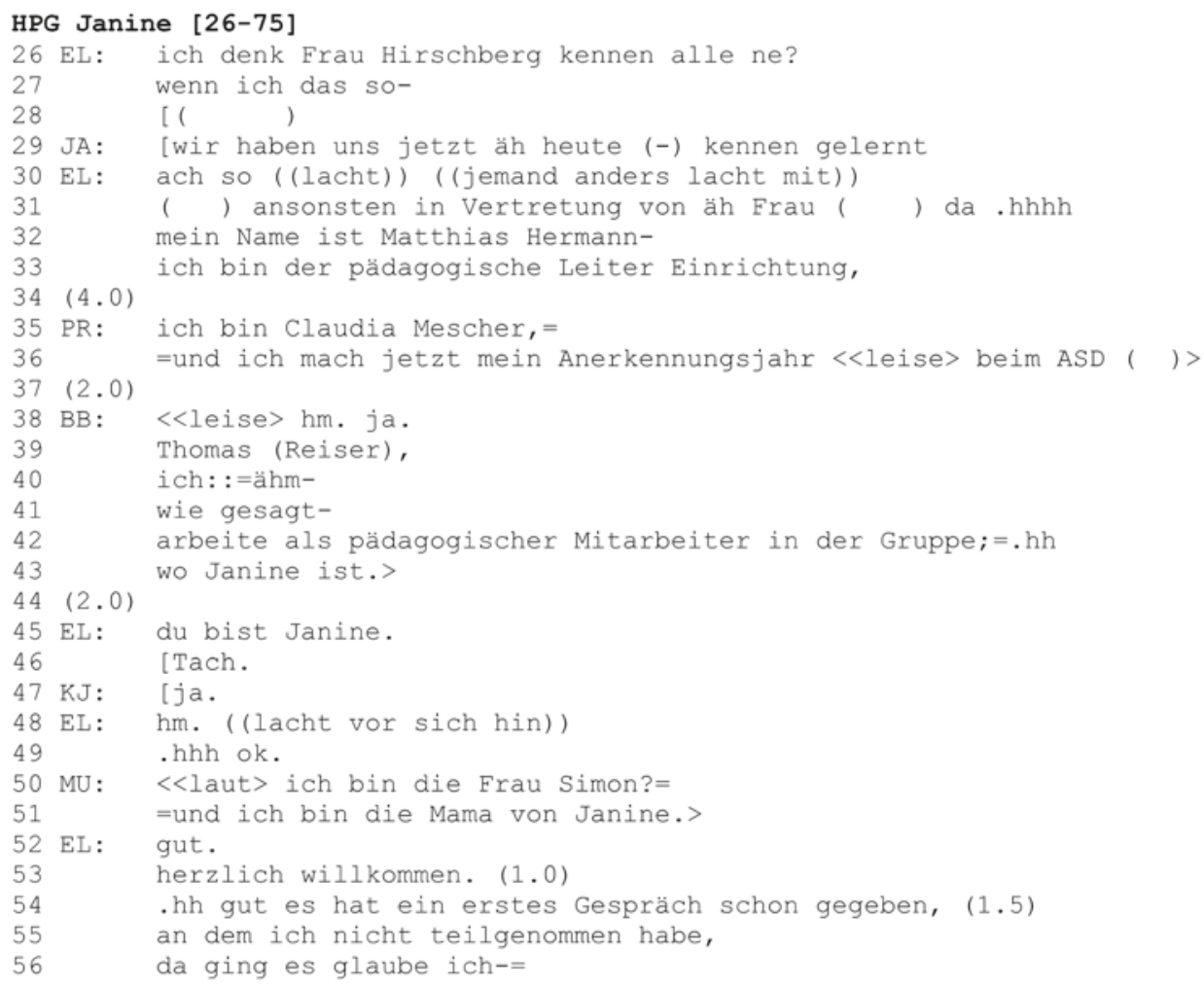

1 Die Konventionen zur Transkription befinden sich im Appendix zu diesem Aufsatz. Die Sprechersiglen bedeuten im Einzelnen EL: Erziehungsleiter/in; JA: Fachkraft Jugendamt; PR: Praktikant/in; BB: Bezugsbetreuer/in; KJ: Kind/Jugendliche/r; MU: Mutter. 


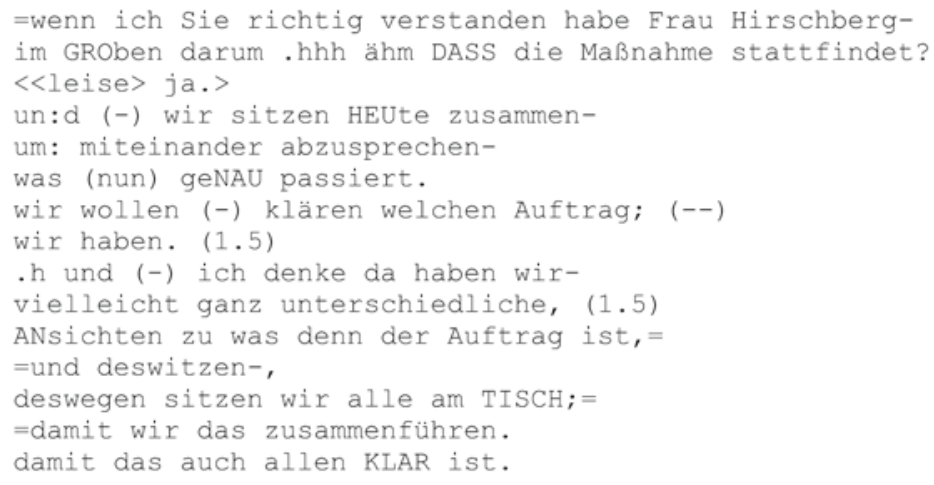

Das hier vorgestellte, etwas längere Exzerpt zerfällt in zwei Teile: eine Vorstellungsrunde unter den Anwesenden (Z 26-53) sowie Erläuterungen zum Sinn und Zweck des Hilfeplangesprächs aus Sicht von EL (Z 54-75). Beide Aktivitäten dienen zuerst und vor allem der Etablierung des vorliegenden Settings. Offenkundige Aspekte von Moral oder des Moralisierens sind nicht evident. Bei genauerer Betrachtung geben sich jedoch diverse Äußerungsformen zu erkennen, über die sich die moralische Dimension der hier vorgestellten Aktivitäten näher erschließt.

\subsubsection{Interaktive Teilhabe}

Ein erster Gesichtspunkt der Analyse zielt auf den Zusammenhang von Interaktion und Moral. Mit der Vorstellungsrunde werden zunächst die Identitäten der Anwesenden füreinander sichtbar gemacht. Damit wird eine grundlegende Bedingung der Teilnahme an Kommunikation und des gemeinsamen Handelns erfüllt (vgl. Krappmann 1969). Insbesondere in größeren Gesprächsrunden, deren Teilnehmende sich untereinander nicht oder nur teilweise kennen, stellt sich die Frage, in welcher Form die Vorstellungsrunde realisiert werden soll, welche Erwartungen dabei berücksichtigt werden müssen und wie der Verschiedenheit der Beteiligten Rechnung zu tragen ist. Vor diesem Hintergrund fällt zunächst die weitgehende Gleichförmigkeit der Darstellungsformen auf. Alle Personen mit Ausnahme von Janine (und JA, die hier irrtümlich als bekannt vorausgesetzt wird) machen sich mit Namen und Rollenbezeichnung untereinander bekannt. Formal wird damit dem Gleichheitsgrundsatz entsprochen: Die Beteiligten erhalten nicht nur (gleiche) Informationen über die Identität des je anderen, sondern darüber hinaus auch die Anerkennung als legitime Teilnehmende am Gespräch.

Neben Aspekten der interaktiven Gleichbehandlung sind in diesem Exzerpt verschiedene Dimensionen einer kommunikativen Ethik bedeutsam (vgl. Bülow 1971). Angesprochen sind Fairnessregeln des kommunikativen Umgangs mit- und untereinander, die unter den Teilnehmenden gleiche Teilhabechancen gewährleisten können. Diese äußern sich in den vorliegenden Gesprächsdaten beispielhaft in Form längerer Pausen zwischen den einzelnen turns (Z 34, 37, 44), in der Unterstützung Janines Vorstellung durch EL 
(Z 45 ff.), der Ermunterung zur Übernahme eines turns (Z 49:. hhh ok), durch auffallende Zurückhaltung (leises Sprechen) in der Ausführung einzelner turns (Z 36, $38 \mathrm{ff}$.) sowie in der abschließenden Anerkennung der einzelnen Beiträge durch EL (Z 52: gut.). Mit den hier angeführten sprachlichen Formen wird nicht nur der „Verweigerung oder Missachtung des Rechts auf gleiche physische und symbolische Integrität" (Schmidt 2000, S.388) entgegengewirkt, sondern einer Erwartungsstruktur Rechnung getragen, die den Beteiligten gleiche Berücksichtigungschancen gewährt.

\subsubsection{Informative Teilhabe}

Eine vergleichbare Situation ergibt sich im Hinblick auf die zweite Teilaktivität, mit der EL Sinn und Zweck des Zusammentreffens erläutert und die Voraussetzungen für das weitere Vorgehen benennt (Z 54-75). Rein sachlich zielt diese Aktivität auf die Etablierung eines gemeinsamen Gesprächsrahmens, der das Zurückliegende (Z 54: es hat ein erstes Gespräch schon gegeben) mit zukünftigen Maßnahmen (Z 63f.: wir wollen (-) klären welchen Auftrag, wir haben.) verknüpft. Auch hier liegt der Bezug zur Moral darin begründet, dass den Anwesenden auf dem Wege geteilter Informationen gleiche Teilhabechancen eingeräumt werden. Der Bezug wird von EL dadurch hergestellt, indem er a) den gemeinsamen Ausgangspunkt für das weitere Vorgehen markiert (Z 57: DASS die Maßnahme stattfindet), b) das weitere Vorgehen als gemeinsame Aktivität definiert ( $Z$ 60: wir; Z 61: miteinander; Z 69: wir alle), c) den Anwesenden gleichwohl abweichende Standpunkte konzediert (Z 65 ff.: ich denke da haben wir-vielleicht ganz unterschiedliche, (1.5) Ansichten) und d) für das weitere Vorgehen Verfahrenstransparenz reklamiert (Z 71: damit das auch allen KLAR ist).

Trotz der Betonung gemeinsamen Vorgehens verhält es sich so, dass der weitere Gesprächsverlauf von EL einseitig definiert und abgesteckt wird. Dieser Sachverhalt ist dem Sprecher jedoch durchaus bewusst, sofern er Anpassungs- und Korrekturbereitschaften signalisiert, mit denen er seine Ausführungen unter Vorbehalt stellt. Beispielsweise räumt er in Bezug auf den Ausgangspunkt des Gesprächs (DASS die Maßnahme stattfindet) einschränkend ein, dass er an dem besagten Gespräch selbst nicht teilgenommen hat (Z 55), seine Inhalte nur vom Hörensagen kennt (Z 56: da ging es glaube ich- =) und Missverständnisse daher nicht auszuschließen sind (Z 57: = wenn ich Sie richtig verstanden habe Frau Hirschberg-). Darüber hinaus gibt EL zu erkennen, dass er die Interessen und Bedürfnisse der anderen mitbedenkt: Neben dem Umstand, dass vielleicht ganz unterschiedliche, (1.5) Ansichten (Z 66f.) darüber bestehen, was der noch näher zu bestimmende Auftrag an die Einrichtung ist, das Ergebnis dieser Zusammenkunft daher noch offen und unbestimmt ist, sind es vor allem die längeren Pausen zwischen (Z 64) und am Ende des turns $(Z$ 72, 74), mit denen EL den Anwesenden Gelegenheit zur turnÜbernahme - und damit zur Artikulation ergänzender, korrigierender oder widersprechender Ansichten bietet.

Obwohl das vorliegende Exzerpt keine ausdrücklichen moralischen Urteile enthält, geben sich gleichwohl moralische Phänomene der Interaktion zu erkennen. Diese betreffen zunächst den Status der Teilnehmenden am Gespräch. Durch formal gleiche Anerkennungs- und Berücksichtigungschancen wird eine Situation etabliert, die den Teilnehmenden formal gleiche Möglichkeiten und Rechte gewährt. Damit werden bestehende 
Status- und Rollenungleichgewichte bis auf weiteres neutralisiert. Moral wird dabei weniger im Sinne retrospektiver Handlungsbewertungen virulent, sondern äußert sich vor allem proaktiv im Sinne der Ermöglichung einer Handlung.

\subsection{Moral im Spannungsfeld zwischen Defizitkonstruktion und Stigmavermeidung}

In diesem Abschnitt rückt ein Phänomen der Moralkommunikation in den Blick, das dadurch auffällt, als es sich der Aufmerksamkeit zu entziehen versucht. Gemeint sind Äußerungen, die unter dem Begriff des „Moralisierens“ zusammengefasst werden können, also die Bezeichnung eines Verhaltens unter dem Gesichtspunkt sittlicher Abweichung. Im Fall „Janine“ liegen moralisierende Handlungsbewertungen durchaus nahe: Ihr Wegbleiben über Nacht lässt sich als Hinweis für sexuelle Frühreife deuten, zumal sich Janine der mütterlichen Fürsorge in einem Maße entzieht, die staatliche Fürsorge ratsam macht. Die Thematisierung dieses Sachverhalts generiert für die Fachkräfte jedoch auch Probleme. Einerseits ist die Beurteilung von Janines Verhalten Auslöser und Rechtfertigung einer staatlichen Intervention, andererseits besteht Gefahr, dass die Erörterung dieses Themas im Beisein der Betroffenen zu unvorhersehbaren Reaktionen oder gar Widerstand führt. Entsprechend vorsichtig formuliert JA:

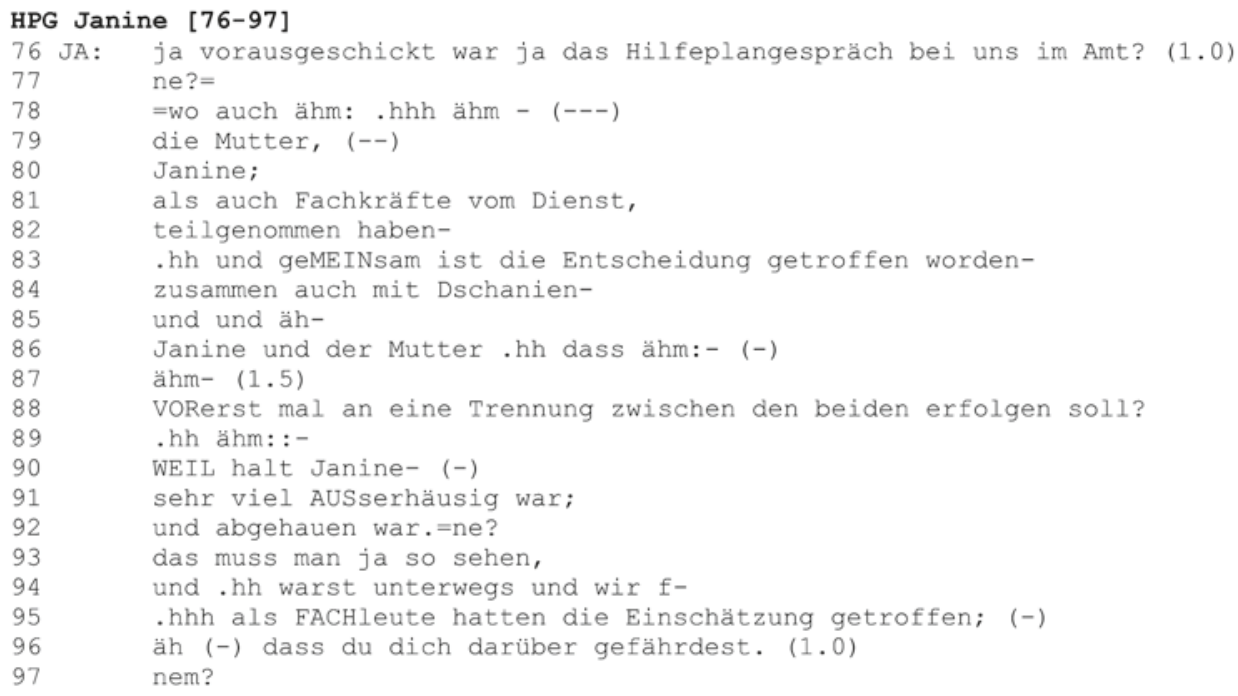

In diesem Exzerpt begründet JA die Unterbringung mit der Außerhäusigkeit und Selbstgefährdung Janines. Dabei fällt auf, dass die von ihr gewählten Begrifflichkeiten den Sachverhalt mit größtmöglicher Neutralität und Zurückhaltung umschreiben (Z 91: AUSserhäusig; Z 92: abgehauen; Z 94: warst unterwegs). Weder wird der Sachverhalt inhaltlich präzisiert, noch werden Fragen der Verantwortlichkeit aufgeworfen. Zudem lassen sich aus der Fallbeschreibung keinerlei Rückschlüsse ziehen, die auf ein Erziehungsversagen der Mutter hindeuten würden. Es wird lediglich konstatiert, dass im Einvernehmen aller Beteiligten VORerst mal eine Trennung zwischen den beiden erfolgen soll? (Z 88). 
Die These gemiedener Moralisierung wird zudem durch verschiedene kommunikative Formate unterstützt, an denen sich die Meidungsabsicht achtungsbedrohender Mitteilungsformen ebenfalls gut ablesen lässt, so beispielsweise, wenn JA den Inhalt der zuvor getroffenen Entscheidung wiederzugeben versucht (Z 86f.:. hh dass ähm:- (-) ähm(1.5)) bzw. über einem angemessenen Begriff für Janines Verhalten sinniert (Z 89f.:.hh ähm:: -WEIL halt Janine). Ihr Zögern ist mithin indikatorisch. Die Konversationsanalyse spricht in diesem Zusammenhang von reluctance markers (Bilmes 1988, S. 173) oder delay devices (Sacks 1987, S. 58), an denen sich die Vermeidungs- oder Abschwächungsabsicht einer (unangenehmen) Mitteilung empirisch manifestiert. Die Notwendigkeit, Janines Situation und Verhalten möglichst genau zu beschreiben, so dass die bis dahin erfolgte Trennung zwischen Mutter und Kind verständlich und nachvollziehbar erscheint, kontrastiert mit dem Wunsch, Formulierungen und Begriffe zu vermeiden, aus denen sich u. U. ein moralischer Vorwurf heraushören lässt. Die Achtung der Person kommt in diesem Exzerpt also dadurch zum Ausdruck, dass die Fachkraft potentiell missachtende Handlungsbewertungen unterdrückt und stigmatisierende Formulierungen unterbleiben. Folge davon ist jedoch eine Umschreibung, welche die Trennung zwar nicht mehr einsichtig zu erklären vermag, jedoch den vorliegenden Verständigungszwecken durchaus genügt.

\subsection{Moral im Kontext der interaktiven Produktion von Verantwortlichkeit}

Nicht immer lassen sich Moralisierungen im Rahmen eines HPG vermeiden, mitunter sind sie sogar erwünscht - so zum Beispiel, wenn die Mitarbeit der Klientel bei der Umsetzung einer Hilfe nicht den Erwartungen der Professionellen genügt. Vordergründig sind solche Äußerungsformen auf Einsicht und Verhaltensänderung angelegt, indem sie an die Verantwortlichkeit der Betroffenen appellieren. Impliziert ist jedoch auch eine Vorstellung moralischen Handelns. Hintergrund der nachfolgenden Episode ist, dass Janine in der Clearingstelle auffällig oft die Nähe von Jungen sucht, Besuch von Jungen in ihrem Zimmer hat (was ihr untersagt ist) oder sich im „Flachbau“ aufhält (was ihr ebenfalls untersagt ist). Vor diesem Hintergrund macht das nachfolgende Statement auf Janines Regelverstoß aufmerksam: 


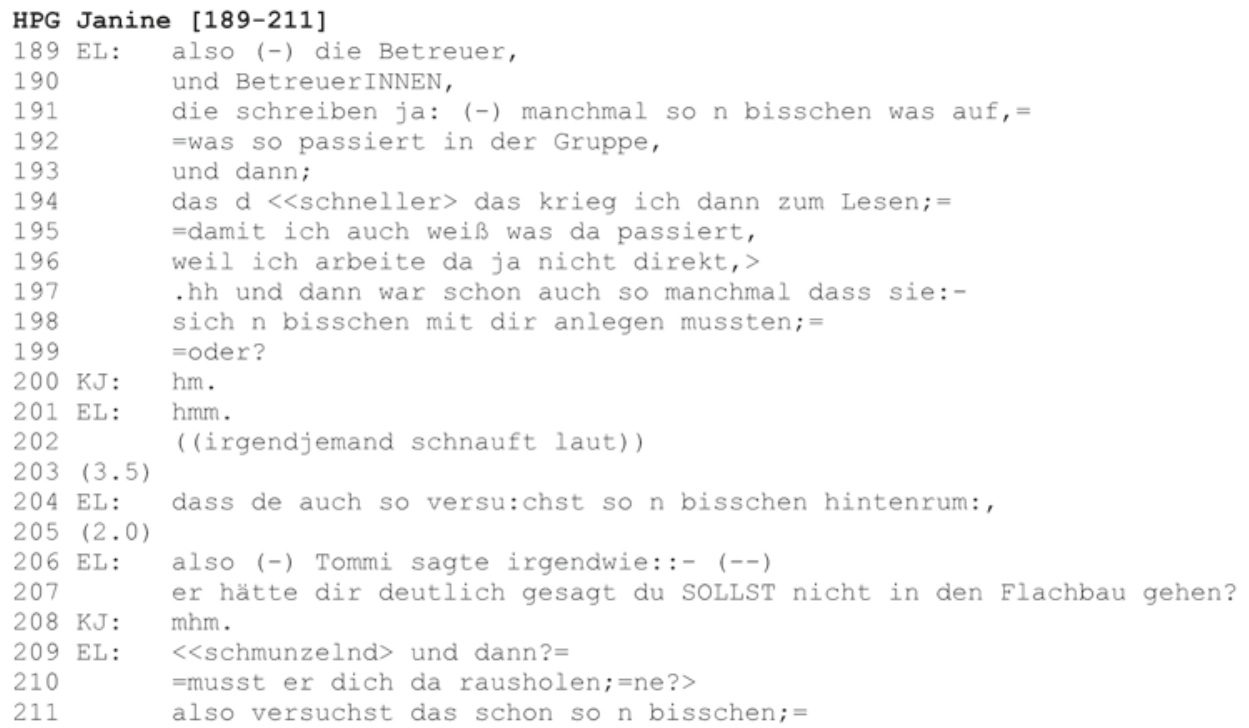

Die Thematisierung von Regelverstößen ist mit moralischen Urteilen eng verwandt. Hier wie dort steht die Beurteilung einer „Objektivität des Sollens“ zur Disposition. Äußerungen dieses Typs sind - um einen Begriff der Konversationsanalyse zu gebrauchen - im hohen Maße inference rich (vgl. Sacks 1992, S.40f.) und entsprechend anfällig für Widerspruch und Konflikt. Wie das vorliegende Exzerptbeispiel zeigt, ist sich EL der Konfliktanfälligkeit moralnaher Kommunikationen durchaus bewusst, weswegen er kommunikative Formate bemüht, die dem Risiko einer Konflikteskalation vorbeugen sollen. Ein erster Aspekt der Konfliktmeidung wird dadurch ersichtlich, dass EL die indirekte Kommunikation präferiert: Der fragliche Sachverhalt wird nicht von BB, der als Beteiligter selbst anwesend ist, zur Sprache gebracht, sondern durch einen nicht direkt beteiligten Dritten. ${ }^{2}$ Als bloßer Übermittler einer die Achtung bedrohenden Mitteilung ist EL daher auch nicht unmittelbar der Adressat für Widerspruch, als solcher auch nicht direkt betroffen, was wiederum die Wahrscheinlichkeit affektgetönter Reaktionen reduziert und zur Versachlichung eines potentiellen Konflikts beitragen kann.

Darüber hinaus fällt in EL's Äußerung der gehäufte Gebrauch von migitativen und diminutiven Sprachformen auf, deren Aufgabe es ist, den Anschuldigungssachverhalt zu entschärfen, so dass er leichter hinnehmbar wird. Verschiedene Abschwächungspartikel (Z 191: manchmal so $n$ bisschen; Z 197: schon auch so manchmal; Z 198: $n$ bisschen; Z 204: auch so versu:chst so $n$ bisschen; Z 206: also (-) Tommi sagte irgendwie:: (-); $\mathrm{Z}$ 211: also versuchst das schon so $n$ bisschen) wirken dabei in zeitlicher (manchmal), sachlicher ( $n$ bisschen) und sozialer Hinsicht (versu:chst) zusammen. Ungeachtet des-

2 Damit rückt diese kommunikative Form in unmittelbare Nähe zum Klatsch (vgl. ausführlich Bergmann 1987), eine kommunikative Gattung, die ebenfalls moralische Sachverhalte thematisiert, definitionsgemäß jedoch über abwesende Dritte, so dass kein direkter Konflikt zu befürchten ist. 
sen bleiben die achtungsbedrohenden Implikationen der Anschuldigungskommunikation virulent. Waren Janines Reaktionen bis zu dieser Stelle im Gespräch durchweg kooperativer Natur, erfolgt im Anschluss an ELs Vorhalt erstmals (empörter) Widerstand:

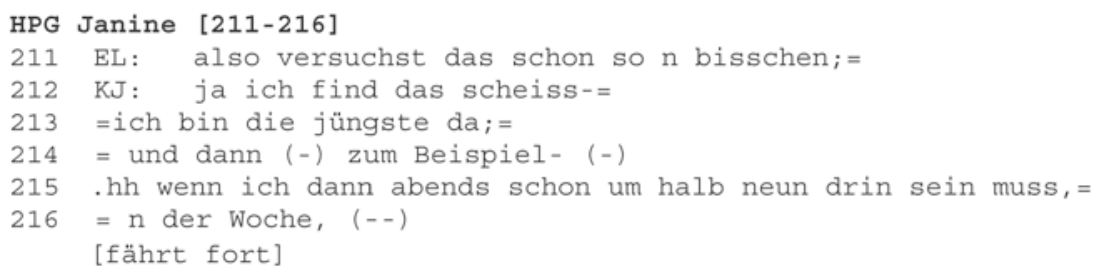

\subsection{Die Moral des Helfens}

Ein letztes Beispiel greift auf die eingangs beschriebene Moralität des Helfens zurück. Demnach ist „Hilfe“ an sich schon Ausdruck moralischen Handelns, zumal sie ohne gleichwertige Gegenleistung auf die Unterstützung hilfebedürftiger Individuen zielt. Im Akt des Helfens drückt sich die Fürsorge für den anderen aus, selbst wenn sie sich in primär wohlfahrtsstaatlichen Kontexten vollzieht. Das nachfolgende Exzerpt ist ein Anwendungsfall dessen und macht die Moral des Helfens unmittelbar evident:

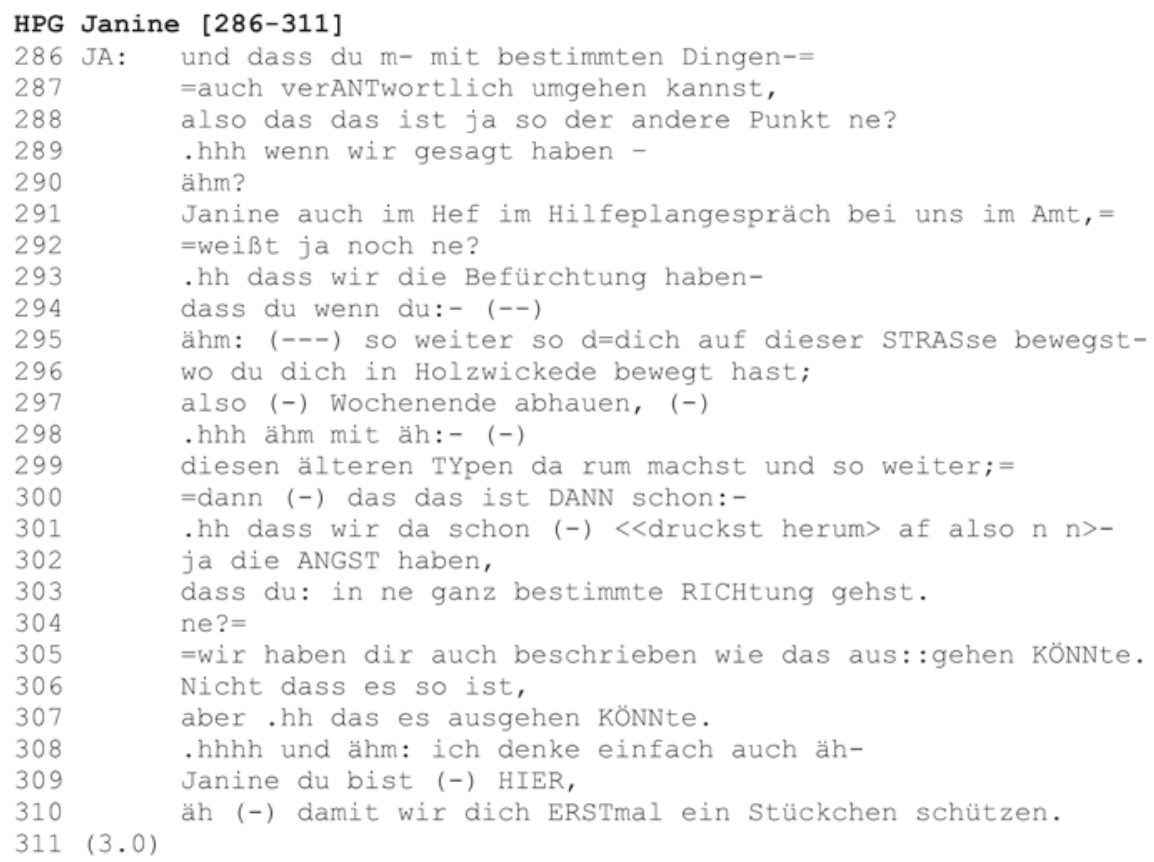

Die Moral des Helfens begründet sich im vorliegenden Fall als Ausdruck von Befürchtung (Z 293) und ANGST (Z 302), vorzugsweise also über eine emotionale Qualität, in der sich der Anlass zur Sorge manifestiert. Auch das Komplement zum Hilfebedarf, 
die Fremdunterbringung, ist deutlich von fürsorglichen Aspekten des Helfens geprägt - dem Schutz des Kindes vor Gefahren für sein Wohlergehen. Entsprechend fasst das vorliegende Statement verschiedene Aspekte der Moralkommunikation in der Beziehung zwischen Professionellen und Klienten zusammen. Obwohl JA den Hilfe auslösenden Sachverhalt hier weit deutlicher expliziert als zuvor (Z $295 \mathrm{ff}$.: so weiter so $d=$ dich auf STRASse bewegst- (...) also (-) Wochenende abhauen, (-).hhh ähm mit äh:- (-) diesen älteren Typen da rum machst und so weiter; =), sind verschiedene Redepartikel (Dehnlaute, Verzögerungen, Pausen, Wort- und Satzabbrüche, hörbares Einatmen, etc.) ebenso wie das Absehen moralisierender Handlungsbewertungen (Z 303: dass du: in ne ganz bestimmte RICHtung gehst) erneut Beleg für die Stigma vermeidenden Intentionen der Professionellen. In anderen, mehr lebensweltlich geprägten Gesprächssettings hätten die hier herangezogenen Kategorien (STRASse, abhauen, älteren Typen da rum machst) eventuell den Schluss nahegelegt, dass es sich bei Janine um ein unmoralisches oder promiskuitives Mädchen handelt. Diese Möglichkeit steht in Form einer Drohung ansatzweise zwar im Raum (Z 294: wenn $\rightarrow$ Z 300: = dann), wird letzten Endes jedoch wieder fallengelassen und nicht mehr weiterverfolgt. Gemäß dem Hinweis in Z 305 (= wir haben dir auch beschrieben wie das aus::gehen KÖNNte) ist dies möglicherweise schon zu einem früheren Zeitpunkt geschehen. Anstatt die Handlung zu verurteilen, besinnt sich JA an dieser Stelle auf das eigentliche Ziel des vorliegenden Settings - der Passung von Hilfebedarf (Selbstgefährdung) und einer diesbezüglichen Hilfe:

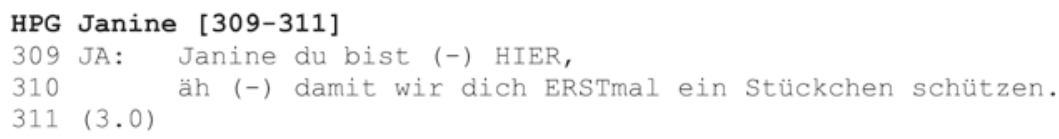

\section{Diskussion}

Ohne Anspruch auf analytische oder systematische Vollständigkeit hatte die vorliegende Studie zum Ziel, Erscheinungsformen von Moral in den Interaktionen zwischen Professionellen und KlientInnen sichtbar zu machen und ihre Bedeutung in Grundzügen zu skizzieren. Dabei hat sich gezeigt, dass Moral als eine die institutionellen Aktivitäten der Sozialen Arbeit umfassende Orientierung aufgefasst werden kann, die nicht nur Aspekte des Beziehungsungleichgewichts zwischen Professionellen und Klienten zu kompensieren vermag, sondern darüber hinaus auch den ethischen Prinzipien professionellen Handelns Ausdruck verschafft. Vor diesem Hintergrund möchte ich die hauptsächlichen Einsichten der vorliegenden Analysen abschließend kurz erörtern.

Ein erster Gesichtspunkt betrifft zunächst die Allgegenwart der Moral. Aus konversationsanalytischer Sicht machen die vorliegenden Untersuchungen deutlich, dass jede Kommunikation im Rahmen der Hilfeplangespräche Aspekte von Moral in sich trägt, aus denen sich Hinweise hinsichtlich der Achtungswürdigkeit einer Person ableiten lassen (vgl. Hydén 1994, 1999). Entsprechend sind ihre Erscheinungsformen ebenso vielfältig wie komplex. Nicht immer zeigt sich Moral in Form einer (retrospektiv) achtenden oder missachtenden Handlungsbewertung explizit und direkt. Weit mehr sind es gerade die unscheinbaren, impliziten und para-sprachlichen Mitteilungsformen, an denen sich die 
Berücksichtigung der Interessen und Bedürfnisse anderer ablesen lässt. Damit wird nicht nur den basalen Voraussetzungen einer kommunikativen Ethik entsprochen, sondern auch den Prinzipien prozeduraler Gerechtigkeit (fair treatment, vgl. Blader und Tyler 2003) Genüge getan, deren Fundament auf dem fortlaufenden Mitverstehen der Interessen und Bedürfnisse anderer ruht.

Ein zweiter Gesichtspunkt berührt die Funktion von Moral. Moral (im Sinne eines Ausdrucks von Achtung) steht synonym für ein Inklusionsgebot, das Mitgliedschaft (vgl. Luhmann 1997) und Solidarität (vgl. Parsons 1972) in sozialen und institutionellen Erwartungszusammenhängen als einen ordnungsbildenden Faktor begreift. Moral zielt auf den Zusammenhalt sozialer Ordnung, was im Hinblick auf Interaktion vor allem Teilhabechancen notwendig macht. Im Fall der Exklusionsvermeidung sind die Voraussetzungen dagegen anders gelagert. Wie die vorliegenden Analysen zeigen, ist das Exklusionsrisiko selbst ein Produkt der institutionellen Aktivität und in Form einer die Hilfe begründenden Defizitkonstruktion in den Interaktionen latent gegenwärtig (Janine als unmoralisches Mädchen, Erziehungsversagen der Mutter). Dieser Sachverhalt ist den institutionellen Akteuren - wie sich an ihrer Kommunikation gut ablesen lässt - durchaus bewusst. In den hier untersuchten Situationen wird die Möglichkeit stigmatisierender Kommunikationen dadurch entschärft, indem die Fachkräfte das defizitäre Verhalten nur insoweit explizieren, wie es zu Verständigungszwecken unbedingt notwendig ist - vorzugsweise mit Hilfe sachlicher Umschreibungen und neutraler Begriffe.

In dieselbe Richtung weist schließlich auch die Moral einer Hilfe, in der sich vor allem die Selbstsicht der Professionellen reflektiert. Soweit sich ihr Handeln nicht nur sachlich, im Sinne einer Leistungserbringung, sondern auch über moralische Ziele legitimiert (Hilfe, Schutz, Sorge um den anderen), spiegelt sich in den Zielen institutioneller Aktivitäten das Gebot der Exklusionsvermeidung ebenfalls wider. In logischer Umkehrung folgt daraus jedoch auch eine reziproke Erwartungsstruktur: Wer sich der Hilfe verweigert oder ihre Umsetzung nicht adäquat unterstützt, setzt sich damit selbst in ein moralisches Unrecht. Folglich zielt die explizite Thematisierung von Moral auf ein Verhalten, das den institutionellen Erwartungen nicht entspricht. In diesem Fall wird die Verantwortung für das betreffende Verhalten explizit der Person zugerechnet. Dieser Situation liegt die Vorstellung eines ,guten“ Klienten zugrunde, der durch sein Verhalten die Absichten der Professionellen unterstützt, während ,schlechte“ Klienten sich den ,guten“Absichten der Professionellen bzw. ihren identitätsgenerierenden Zuschreibungen widersetzen (vgl. Juhila 2003; Urek 2005; Keddell 2011; Solberg 2011).

Moral in der Interaktion zwischen Professionellen und Klienten ist den vorliegenden Analysen gemäß ein ebenso universelles wie flexibel anwendbares Beziehungskonstrukt (vgl. Jayyusi 1984; Goblirsch 2006). Es eignet sich sowohl zur Her- und Sicherstellung von Kooperation wie auch zur Disziplinierung der betroffenen Klientel - was sich wechselseitig nicht unbedingt ausschließt (vgl. Heiner 2007). Entgegen dem herkömmlichen Begriffsverständnis von Moral äußert sich diese jedoch weniger in Form einer expliziten und direkten Handlungsbewertung, sondern fällt weit mehr durch ihre Abwesenheit ins Gewicht. Der Verzicht auf moralische Urteile im Rahmen der Fallkonstitution begrenzt nicht nur das institutionell produzierte Exklusionsrisiko, sondern unterstützt die Inklusion der Betroffenen in das institutionelle Setting, das wesentlich durch die Erwartungen der Professionellen vorherbestimmt ist. Die Wirksamkeit der Moral erweist sich dabei 
an den handlungsermöglichenden Konsequenzen, indem sie der strukturell schwächeren Seite Raum für Einflussnahme und Teilhabe schafft.

Auf diese Weise werden Probleme gelöst, die dadurch entstehen, dass Status und Rolle in institutionellen Kontexten den Beteiligten regelmäßig ungleiche Berücksichtigungschancen bieten. Dieser Gesichtspunkt ist auch deshalb bedeutsam, weil das institutionelle Handeln eigene, für es nützliche Normen kreiert - einschließlich des Rechts auf moralische Beurteilung. Vor diesem Hintergrund unterstützt Moral die Berücksichtigung der Klientel in einem systemischen Erwartungszusammenhang sowie die Umsetzung entsprechender Ziele. Nicht zuletzt tritt sie damit auch der Versachlichung und Objektivierung ihrer Adressaten entgegen. Andererseits treten die Grenzen der Berücksichtigung regelmäßig dort in Erscheinung, wo die institutionelle Zielsetzung zu den Bedürfnissen und Interessen ihrer Klientel mutmaßlich oder explizit in Widerspruch steht. Dies ist beispielsweise dann der Fall, wenn eine Hilfe gegen die Wünsche der Betroffenen nicht mehr weitergeführt wird (vgl. Messmer und Hitzler 2008), wenn aus Sicht der Professionellen Entscheidungen notwendig werden, bei denen mit Widerstand der Betroffenen zu rechnen ist (vgl. Hitzler und Messmer 2010) oder die Spannung zwischen einem normativ gesetzten Partizipationspostulat und den Möglichkeiten seiner Realisierung interaktiv nicht mehr überbrückt werden kann (vgl. Greschke et al. 2010).

Vor dem Hintergrund dieser, aber auch in internationaler Perspektive vergleichbaren Forschungen zu den interaktiven Praxen Sozialer Arbeit (vgl. Hall et al. 2003; Hall et al. 2006; Hitzler und Messmer 2008) verdichtet sich der Eindruck, dass die empirisch vorfindbare Moral professionellen Handelns mehr ein Gemisch der Eigenlogiken der Disziplin und weniger ein Gebot sittlichen Handelns ist. Wer beispielsweise aufgrund rechtlicher, ökonomischer und politischer Vorgaben fristgerecht zu einer fachlich vertretbaren und nachvollziehbaren Entscheidung kommen muss, kann die institutionellen Determinanten der Entscheidungsfindung nur bedingt mit den Erwartungen der Entscheidungsbetroffenen synchronisieren. Nicht nur im Hinblick auf die Achtungswürdigkeit der Person, sondern auch hinsichtlich der Berücksichtigung ihrer Wünsche und Interessen sind die Grenzen mithin enger gezogen, als es der aktuelle Diskurs über moralisch wertschätzende Orientierungen in der Sozialen Arbeit suggeriert. 


\section{Transkriptionskonventionen und Sprechersiglen}

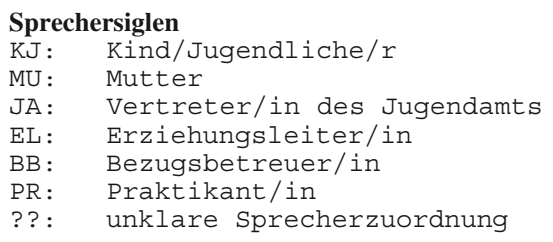

\section{Transkriptionskonventionen in Anlehnung an GAT}

Tonhöhenbewegung

? hoch steigend

mittel steigend

gleichbleibend

mittel fallend

tief fallend

Sonstige Konventionen

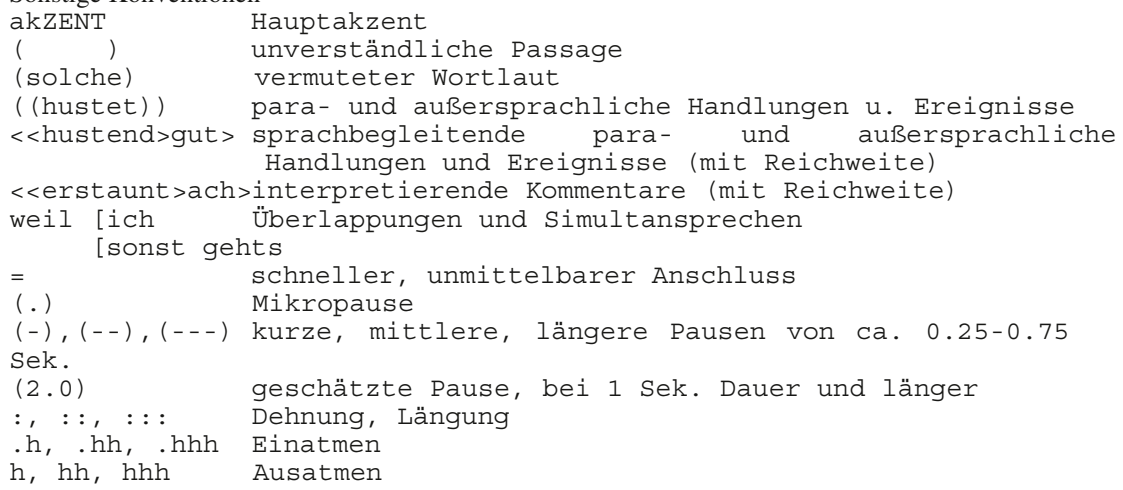

\section{Literatur}

AvenirSocial. (2010). Berufskodex Soziale Arbeit Schweiz. Ein Argumentarium für die Praxis der Professionellen. Bern: AvenirSocial.

Beetz, M. (2009). Was können Soziologen von Moral verstehen? Gesellschaftliche Praxisfelder und ihre moralischen Kompetenzerfordernisse. Berliner Journal für Soziologie, 19, 248-267.

Bergmann, J. (1985). Flüchtigkeit und methodische Fixierung sozialer Wirklichkeit: Aufzeichnungen als Daten der interpretativen Soziologie. In W. Bonß \& H. Hartmann (Hrsg.), Entzauberte Wissenschaft: Zur Relativität und Geltung soziologischer Forschung (S. 299-320). Göttingen: Schwarz.

Bergmann, J. (1987). Klatsch: zur Sozialform der diskreten Indiskretion. Berlin u. a.: de Gruyter.

Bergmann, J., \& Luckmann Th. (1999). Moral und Kommunikation. In J. Bergmann \& Th. Luckmann (Hrsg.), Kommunikative Konstruktion von Moral. Bd. 1: Struktur und Dynamik der Formen moralischer Kommunikation (S. 15-36). Opladen: Westdeutscher.

Bilmes, J. (1988). The concept of preference in conversation analysis. Language and Society, 17, $161-181$.

Blader, S. E., \& Tyler, T. R. (2003). A four-component model of procedural justice: Defining the meaning of a ,fair“ process. Personality and Social Psychology Bulletin, 29, 747-758.

Bülow, E. (1971). Kommunikative Ethik. Düsseldorf: Schwann.

Dungs, S., Gerber, U., Schmidt, H., \& Schmidt, R. (Hrsg.). (2006). Soziale Arbeit und Ethik im 21. Jahrhundert: ein Handbuch. Leipzig: Evangelische Verlagsanstalt. 
Durkheim, É. (1973). Erziehung, Moral und Gesellschaft. Neuwied: Luchterhand.

Durkheim, É. (1976). Der Regeln der soziologischen Methode. Neuwied: Luchterhand.

Eisenmann, P. (2006). Werte und Normen in der Sozialen Arbeit. Stuttgart: Kohlhammer.

Friesinger, T. (2007). Ethik der Sozialen Arbeit: Eine Fallbearbeitung mit Ausschnitten aus der praktischen Diskursethik von Jürgen Habermas, der Kommunikationstheorie von Niklas Luhmann sowie unter dem Aspekt der eigenen ethischen Grundpositionen. Norderstedt: GRIN.

Fuchs, P. (2004). Die Moral des Systems Sozialer Arbeit - systematisch. In R. Merten \& A. Scherr (Hrsg.), Inklusion und Exklusion in der Sozialen Arbeit (S. 17-32). Wiesbaden: VS-Verlag für Sozialwissenschaften.

Gängler, H. (2011). Hilfe. In H.-U. Otto \& H. Thiersch (Hrsg.), Handbuch Soziale Arbeit (S. 609618), München: Reinhardt.

Goblirsch, M. (2006). Zur kommunikativen Herstellung von Identität und Moral. In P. Cloos \& W. Thole (Hrsg.), Ethnografische Zugänge. Professions- und adressatenbezogene Forschung im Kontext von Pädagogik (S. 157-168). Wiesbaden: VS-Verlag für Sozialwissenschaften.

Greschke, H., Klingler, B., \& Messmer, H. (2010). Praxis im Modellprogramm. Fallstudien zum Hilfeplangespräch. In S. Albus et al. (Hrsg.), Abschlussbericht der Evaluation des Bundesmodellprogramms „Wirkungsorientierte Jugendhilfe“. Schriftenreihe des ISA zur Qualifizierung der Hilfen zur Erziehung (S. 62-104). Münster: Waxmann

Goffman, E. (1971). Relations in public. Microstudies of the public order. New York: Basic Books.

Gruber, H.-G. (2005). Ethisch denken und handeln: Grundzüge einer Ethik der sozialen Arbeit. Stuttgart: Lucius \& Lucius.

Hall, Ch., Parton, N., Juhila, K., \& Pösö, T. (Hrsg.). (2003). Constructing clienthood in social work and human services. London u. a.: Kingsley.

Hall, Ch., Sarangi, S., \& Slembrouck, S. (2006). Language practices in social work: Categorisation and accountability in child welfare. London: Routledge.

Heiner, M. (2007). Soziale Arbeit als Beruf. Fälle - Felder - Fähigkeiten, München: Reinhardt.

Hitzler, S., \& Messmer, H. (2008). Gespräche als Forschungsgegenstand in der Sozialen Arbeit. Zeitschrift für Pädagogik, 54, 244-260.

Hitzler, S., \& Messmer, H. (2010). Group decision making in child welfare and the pursuit of participation. Qualitative Social Work, 9, 205-226.

Hitzler, S., \& Messmer, H. (2011). Konversationsanalyse. In G. Oelerich \& H.-U. Otto (Hrsg.), Empirische Forschung und Soziale Arbeit. Ein Lehrbuch (S. 307-311). Wiesbaden: VS-Verlag für Sozialwissenschaften.

Horster, D. (2011). Moralisches Urteilen und Handeln. In M. Haase, S. Mirkovic, \& O. Schumann (Hrsg.), Ethics Education. Unternehmens- und Wirtschaftsethik in der wirtschaftswissenschaftichen Ausbildung (S. 17-44). München: Rainer Hampp.

Hughes, E. (1965). Professions. In K. Lynn (Hrsg.), The professions in America (S. 1-14). Boston: Mifflin.

Hydén, L. (1994). The social worker as a moral worker. Applying for money - the moral encounter between social workers and clients. In B. Gunnarsson, P. Linell, \& B. Nordberg, (Hrsg.), Text and talk in professional contexts (S. 187-199). Uppsala: ASLA.

Hydén, L. (1999). Talk about money. Studying the interaction between social worker and client. International Journal of Social Welfare, 8, 143-154.

Jayyusi, L. (1984). Categorization and the moral order. Boston u. a.: Routledge.

Keddell, E. (2011). Reasoning processes in child protection decision making: Negotiating moral minefields and risky relationships. British Journal of Social Work, 41, 1251-1270.

Krappmann, L. (1969). Soziologische Dimensionen der Identität. Strukturelle Bedingungen für die Teilnahme an Interaktionsprozessen. Stuttgart: Klett-Cotta.

Kuhrau-Neumärker, D. (2005). „War das o.k.?" Moralische Konflikte im Alltag Sozialer Arbeit: Einführung in die Berufsethik. Münster: Waxmann.

Lob-Hüdepohl, A. (Hrsg.). (2007). Ethik Sozialer Arbeit: ein Handbuch. Paderborn: Schöningh. 
Luhmann, N. (1978). Soziologie der Moral. In N. Luhmann \& St. H. Pfürtner (Hrsg.), Theorietechnik und Moral (S. 8-116). Frankfurt a. M.: Suhrkamp.

Luhmann, N. (1984). Soziale Systeme. Grundriss einer allgemeinen Theorie. Frankfurt a. M.: Suhrkamp.

Luhmann, N. (1989). Paradigm Lost: Über die ethische Reflexion der Moral. Frankfurt a. M.: Suhrkamp.

Luhmann, N. (1997). Die Gesellschaft der Gesellschaft. 2 Bd. Frankfurt a. M.: Suhrkamp.

Jaspers, K. (1973). Philosophie II. Existenzerhellung, Berlin: Springer.

Juhila, K. (2003). Creating a ,bad‘ client: Disalignment of institutional identities in social work interaction. In Ch. Hall, N. Parton, K. Juhila, \& T. Pösö (Hrsg.), Constructing clienthood in social work and human services (S. 83-95). London: Kingsley.

Martin, E. (2007). Sozialpädagogische Berufsethik. Auf der Suche nach dem richtigen Handeln. 2. überarb. Aufl. Weinheim: Juventa.

Messmer, H. (2012). Interaktion und Kommunikation in der Sozialen Arbeit - Die Methode der Gesprächsanalyse im Spannungsfeld von kategorien- und gegenstandsgeleiteter Forschung. In D. Gredig \& S. Schnurr (Hrsg.), Forschen in der Sozialen Arbeit - Exemplarische Antworten auf typische methodische Herausforderungen (S. 84-112). Baltmannsweiler: Schneider.

Messmer, H., \& Hitzler, S. (2008). Die Hilfe wird beendet werden hier - Prozesse der Deklientifizierung im Hilfeplangespräch aus gesprächsanalytischer Sicht. neue praxis, 38, 166-187.

Messmer, H., \& Hitzler, S. (2011). Interaktion und Kommunikation in der Sozialen Arbeit - Fallstudien zum Hilfeplangespräch. In G. Oelerich \& H.-U. Otto (Hrsg.), Empirische Forschung und Soziale Arbeit. Ein Lehrbuch (S. 51-64). Wiesbaden: VS-Verlag für Sozialwissenschaften.

Parsons, T. (1972). Commentary on Clark. In A. Effrat (Hrsg.), Perspectives in political sociology (S. 299-308). Indianapolis.

Sacks, H. (1987). On the preferences for agreement and contiguity in sequences in conversation. In G. Button \& J. R. E. Lee (Hrsg.), Talk and social organization (S. 54-69). Clevedon: Multilingual Matters.

Sacks, H. (1992). Lectures on conversation. 2 Bd. (hrsg. von G. Jefferson). Oxford: Blackwell Publishing.

Schmidt, V. H. (2000). Ungleichheit, Exklusion und Gerechtigkeit. Soziale Welt, 51, 383-400.

Selting, M., et al. (2009). Gesprächsanalytisches Transkriptionssystem (GAT2). Gesprächsforschung, 10, 353-402.

Simmel, G. (1892/1893). Einleitung in die Moralwissenschaft. Eine Kritik der ethischen Grundbegriffe. Neudruck Aalen: Scientia-Verlag.

Solberg, J. (2011). Activation encounters: Dilemmas of accountability in construction clients as ,knowledgeable‘. Qualitative Social Work, 10, 381-398.

Stichweh, R. (2004). Wissen und die Profession in einer Organisationsgesellschaft. Verfügbar unter: http://www.unilu.ch/files/19stwprofessionen.pdf. Zugegriffen: 20. Dez. 2011.

Tarkian, T. (2009). Moral, Normativität und Wahrheit. Zur neueren Debatte um Grundlagenfragen der Ethik. Paderborn: Mentis.

Thiersch, H. (2011). Moral und Soziale Arbeit. In H.-U. Otto \& H. Thiersch (Hrsg.), Handbuch Soziale Arbeit (S. 968-979). 4., völlig neu bearb. Aufl. München: Reinhardt.

Urek, M. (2005). Making a case in social work: The construction of an unsuitable mother. Qualitative Social Work, 4, 451-467.

Weber, M. (1934/2010). Die protestantische Ethik und der Geist des Kapitalismus. Hrsg. und eingeleitet von Dirk Kaesler. München: Beck.

Wiesner, R. (2000). SGB VIII. Kinder und Jugendhilfe. München: Beck. 